\title{
Non-Bragg band gaps in 1D metamaterial aperiodic multilayers
}

Juan A. Monsoriu

jmonsori@fis.upv.es

\section{Ricardo A. Depine \\ Enrique Silvestre}

\author{
Departamento de Física Aplicada, Universidad Politécnica de Valencia, E-46022 Valencia, Spain
}

Departamento de Física, Universidad de Buenos Aires, C1428EHA Buenos Aires, Argentina

\begin{abstract}
We investigate the existence of non-Bragg band gaps in $1 \mathrm{D}$ aperiodic photonic structures, namely the Fibonacci and Thue-Morse lattices combining ordinary positive index materials and dispersive metamaterials. Both structures present new band gaps which, in contrast with the usual Bragg gaps, are not based on interference mechanisms. One of these non-Bragg gaps, called zero- $\bar{n}$ gap and corresponding to zero (volume) averaged refractive index, has been reported to be present in Fibonacci lattices. In this paper we extend this result to other aperiodic systems, showing the existence of a zero- $\bar{n}$ gap also in Thue-Morse lattices. Furthermore, we show that these systems can also support two polarization-selective non-Bragg gaps: the zero permeability, and the zero permittivity gaps. Some distinctive aspects of these gaps are outlined and the impact on the photonic spectra produced by the level of the generation of the aperiodic structure is analyzed. [DOI: 10.2971/jeos.2007.07002]
\end{abstract}

Keywords: Fibonacci, Thue-Morse, metamaterial, negative refractive index

\section{Introduction}

In recent years the study of periodic dielectric structures, photonic crystals (PCs), has been a subject of growing interest due to their panoply of applications. The most relevant property of PCs is the possibility of generating photonic band gaps (PBGs) for light propagation in certain geometries [1], analogous to the electronic band gaps of a semiconductor. This effect has been observed in both one-, two-, and three-dimensional structures in the form of absence of light propagation for specific sets of frequencies (see Ref. [2], and references therein). The simplest one-dimensional (1D) PC is the well known Bragg mirror, which is a periodic structure consisting of two different alternating layers of conventional dielectric materials [3]. The Fourier spectrum of this infinite periodic multilayer shows a discrete distribution (equidistant well-defined Bragg peaks) corresponding to the reciprocal lattice vectors.

On the other hand, disordered dielectric media (exhibiting a continuous Fourier spectrum) have also attracted the attention of researchers because they show several interesting properties such as the existence of PBGs with some strong resonances, which can localize light very effectively [4].

Within the intermediate regime between complete order and disorder, aperiodic dielectric multilayers following a deterministic sequence also display characteristic spectral properties not present in either of these extreme cases. Fractal Cantor [5]-[7], Fibonacci $[8,9]$, and Thue-Morse $[10,11]$ systems are some of the most common examples of aperiodic multilayers having, additionally, different characteristics. Fibonacci and Cantor multilayers present a discrete Fourier spectrum characterized by self-similar Bragg peaks. However, from a structural viewpoint, Fibonacci and fractal Cantor lattices belong to two different types of aperiodic systems: while Fibonacci structures are composed of building blocks exhibiting two incommensurate periods (i.e., it is quasiperiodic), in fractal Cantor lattices the resulting building blocks are mutually commensurate by construction [12]. On the other hand, Thue-Morse structures present a continuous Fourier spectrum with discrete singularities, so it can be interpreted essentially as a discrete distribution [13]. For this reason, a Thue-Morse lattice is not considered a quasiperiodic but a deterministic aperiodic system.

Almost every natural material has been used to construct periodic and aperiodic PCs. After the introduction of negative refractive index materials by Veselago [14] in 1968, the recent fabrication of metamaterials (MMs) has drawn renewed interest in them [15]. MMs with negative refraction index are artificially constructed composites exhibiting a negative electric permittivity together with a negative magnetic permeability in the same frequency range. It has been shown that periodic PCs made with alternating layers of MM and ordinary dielectric materials can lead to a new type of photonic band gaps which are not based on interference mechanisms [16, 17]. The first kind of non-Bragg gap arises naturally when the volume average refractive index, $\bar{n}$, of the multilayer equals zero [16]. The second kind appears at frequencies where either the magnetic permeability, $\mu$, or the electric permittivity, $\epsilon$, of the MM is zero [17]. Both kind of non-Bragg gaps are scale-length invariant and very robust against disorder. 
In this paper we investigate the light transmission through 1D aperiodic PCs containing MMs with special attention to the existence of non-Bragg band gaps. Some papers on aperiodic distributions of MMs have been recently published [18]-[21]. Zero $-\bar{n}$ band gaps in Fibonacci stacks of MMs and conventional dielectric materials were investigated in [20, 21]. However, to our knowledge, zero- $\bar{n}$ gaps in other aperiodic systems have not been reported so far. Furthermore, we have found that zero- $\mu$ or zero- $\epsilon$ band gaps arise in frequency ranges omitted in the previous references [20].

This paper is organized as follows. In Section 2 we review the conventional transfer matrix method for the calculation of photonic spectra of a multilayer structure including both electric and magnetic materials. In Section 3 we study the spectral properties of some 1D aperiodic PCs containing MMs. We perform the analysis for two particular cases, namely the Fibonacci and Thue-Morse lattices. Finally, we outline the main results in Section 4. An $\exp (-i \omega t)$ time-dependence is implicit throughout the paper, with $\omega$ the angular frequency, $t$ the time, and $i=\sqrt{-1}$.

\section{THE TRANSFER MATRIX METHOD}

Let us consider the optical propagation through a 1D multilayer combining dielectric materials and MMs. We use the conventional transfer matrix method (see for instance, Ref. [22]) to calculate the photonic spectra of this structure. Although it is a well-known technique, we revise it here for the case when both electric and magnetic materials are considered. Let us consider the scattering at the $j$ th interface between two successive layers whose position, without loss of generality, has been taken as $y=0$. The function $f(x, y)$ represents the $z$-directed component of the electric field for the TE-polarization case (electric field parallel to the layers) and the $z$-directed component of the magnetic field for the TMpolarization case (magnetic field parallel to the layers). This function can be expressed as a combination of forward $\left(f_{j}^{+}\right)$ and backward $\left(f_{j}^{-}\right)$plane waves, $f_{j}=f_{j}^{+}+f_{j}^{-}, f_{j}^{ \pm}(x, y)=$ $A_{j}^{ \pm} \operatorname{expi}\left(k_{x} x \pm k_{j y} y\right)$, where $k_{j y}^{2}=k_{j}^{2}-k_{x}^{2}$, and $k_{j}=\omega n_{j} / c$ is the wave number in the $j$ th medium with refractive index $n_{j}=\sqrt{\epsilon_{j}} \sqrt{\mu_{j}} . A_{j}^{ \pm}$are integration constants to be determined by applying the standard electromagnetic boundary conditions at the interface. These conditions may be written as a $2 \times 2$ linear system of equations given by

$$
\left(\begin{array}{cc}
1 & 1 \\
\frac{k_{j-1 y}}{\sigma_{j-1}} & -\frac{k_{j-1 y}}{\sigma_{j-1}}
\end{array}\right)\left(\begin{array}{c}
A_{j-1}^{+} \\
A_{j-1}^{-}
\end{array}\right)=\left(\begin{array}{cc}
1 & 1 \\
\frac{k_{j y}}{\sigma_{j}} & -\frac{k_{j y}}{\sigma_{j}}
\end{array}\right)\left(\begin{array}{c}
A_{j}^{+} \\
A_{j}^{-}
\end{array}\right)
$$

where $\sigma_{j}=\mu_{j}$ for TE polarization or $\sigma_{j}=\epsilon_{j}$ for TM polarization. In matrix notation

$$
\begin{gathered}
\left(\begin{array}{c}
A_{j-1}^{+} \\
A_{j-1}^{-}
\end{array}\right)=D_{j-1}^{-1} D_{j}\left(\begin{array}{c}
A_{j}^{+} \\
A_{j}^{-}
\end{array}\right), \\
D_{j}=\left(\begin{array}{cc}
1 & 1 \\
\frac{k_{j y}}{\sigma_{j}} & -\frac{k_{j y}}{\sigma_{j}}
\end{array}\right) .
\end{gathered}
$$

The matrix $D_{j-1}^{-1} D_{j}$ is referred to as the transfer matrix at the $j$ th interface. After crossing interface $j$, the plane waves prop- agate through the $j$ th layer until the next interface at the distance $d_{j}$, where the fields satisfy $\tilde{f}_{j}^{ \pm}=\exp \left( \pm i k_{j y} d_{j}\right) f_{j}^{ \pm}$. In matrix form

$$
\begin{gathered}
\left(\begin{array}{c}
A_{j}^{+} \\
A_{j}^{-}
\end{array}\right)=P_{j}\left(\begin{array}{c}
\tilde{A}_{j}^{+} \\
\tilde{A}_{j}^{-}
\end{array}\right), \\
P_{j}=\left(\begin{array}{cc}
\exp \left(-i k_{j y} d_{j}\right) & 0 \\
0 & \exp \left(+i k_{j y} d_{j}\right)
\end{array}\right) .
\end{gathered}
$$

The matrix $P_{j}$ is known as the wave propagation matrix. The iterative application of the transfer and propagation matrices may be used to solve the general problem of transmission through $N$ layers. The first step is

$$
\left(\begin{array}{c}
A_{0}^{+} \\
A_{0}^{-}
\end{array}\right)=D_{0}^{-1} D_{1}\left(\begin{array}{c}
A_{1}^{+} \\
A_{1}^{-}
\end{array}\right)=D_{0}^{-1} D_{1} P_{1} D_{1}^{-1} D_{2}\left(\begin{array}{c}
A_{2}^{+} \\
A_{2}^{-}
\end{array}\right) \text {, }
$$

which may be easily generalized to yield

$$
\left(\begin{array}{c}
A_{0}^{+} \\
A_{0}^{-}
\end{array}\right)=M\left(\begin{array}{c}
A_{N+1}^{+} \\
A_{N+1}^{-}
\end{array}\right)
$$

$$
M=D_{0}^{-1}\left(\prod_{j=1}^{N} D_{j} P_{j} D_{j}^{-1}\right) D_{N+1}
$$

being $j=0$ and $j=N+1$ the incident and transmission media (air in our case). Because no backward plane wave can be found on the right side of the multilayer, $A_{N+1}^{-}=0$. The reflection and transmission coefficients are given by

$$
\begin{gathered}
R=\frac{\left|A_{0}^{-}\right|^{2}}{\left|A_{0}^{+}\right|^{2}}=\frac{\left|M_{21}\right|^{2}}{\left|M_{11}\right|^{2}}, \\
T=\frac{\left|A_{N+1}^{+}\right|^{2}}{\left|A_{0}^{+}\right|^{2}}=\frac{1}{\left|M_{11}\right|^{2}},
\end{gathered}
$$

respectively.

\section{N U MERICAL RES ULTS}

Let us now consider a quasiperiodic multilayer based on the Fibonacci sequence. The Fibonacci numbers, $F_{j}$ with $j=$ $0,1,2, \ldots$, are characterized by the relation $F_{j+1}=F_{j}+F_{j-1}$, with $F_{0}=0$ and $F_{1}=1$, so $\left\{F_{j}\right\}=\{0,1,1,2,3,5,8,13, \ldots\}$. Hence, each number in the sequence is just the sum of the preceeding two. In a similar way, we have considered the Fibonacci sequence based on a recursive relation, $D_{j+1}=$ $\left\{D_{j}, D_{j-1}\right\}$ for $j>1, D_{1}=\{A\}, D_{2}=\{A B\}, D_{3}=\{A B A\}$, $D_{4}=\{A B A A B\}$, and so on. Note that the sequence at generation level $S, D_{S}$ contains $F_{S}$ 'A' layers and $F_{S-1}$ 'B' layers.

The deterministic Thue-Morse sequence is recursively constructed as $G_{j+1}=\left\{G_{j}, \tilde{G}_{j}\right\}$ for $j>1$ and $G_{1}=\{A\}$. $\tilde{G}_{j}$ is obtained from $G_{j}$ by interchanging 'A' and 'B', so $G_{2}=\{A B\}$, $G_{3}=\{A B B A\}, \ldots$ The 'A' or ' $\mathrm{B}^{\prime}$ number of layers at level $S$ is $2^{S-1}$. 


\begin{tabular}{|c|c|}
\hline $\mathrm{A} B \mathrm{~B}$ & $S=2 \mathrm{AB}$ \\
\hline $\mathrm{ABA}$ & $S=3 \mathrm{~A} B \mathrm{~B} \mid \mathrm{A}$ \\
\hline $\mathrm{AB} A \mathrm{~A} \mathrm{~B}$ & $S=4 \mathrm{AB}|\mathrm{B}| \mathrm{A}|\mathrm{B}| \mathrm{A} \mid \mathrm{B}$ \\
\hline
\end{tabular}

\section{(a) Fibonacci}

FIG. 1 Fibonacci (b) and Thue-Morse (b) aperiodic distributions of metamaterials ' $A$ ' and ordinary positive index materials ' $\mathrm{B}$ '.

Figure 1 shows aperiodic multilayers from the Fibonacci and Thue-Morse sequences, up to $S=5$. In our simulations, ' $\mathrm{A}$ ' represent dispersive $\mathrm{MM}$ layers with effective constitutive parameters given by [16]:

$$
\begin{gathered}
\epsilon_{A}=1+\frac{5^{2}}{0.9^{2}-f^{2}}+\frac{10^{2}}{11.5^{2}-f^{2}} \\
\mu_{A}=1+\frac{3^{2}}{0.902^{2}-f^{2}}
\end{gathered}
$$

where $f$ is the frequency measured in GHz. The same kind of dispersion relations have been used to theoretically predict the existence of the zero-n gap in periodic multilayers, later verified in recent experimental results [23]. The ' $\mathrm{B}$ ' layers are the usual positive index materials, air in our case $\left(\epsilon_{B}=\mu_{B}=1\right)$. The widths of the $\mathrm{MM}$ and air layers are $d_{A}=6 \mathrm{~mm}$ and $d_{B}=12 \mathrm{~mm}$, respectively.

In Figure 2 we compare the photonic spectra of the Fibonacci and Thue-Morse multilayers for normal incidence $\left(\theta=0^{\circ}\right)$ and generation levels from 2 to 10 . The gray-code bar gives the reflection coefficient, $R$, of these structures, being $R=1$ ( $T=0)$ in white regions and $R=0(T=1)$ in black regions. The frequency dependence of effective parameters $\epsilon_{A}$ and $\mu_{A}$ is shown in Figure 2c, becoming zero at frequency values 3.787 and $3.133 \mathrm{GHz}$, respectively. (a) Fibonacci

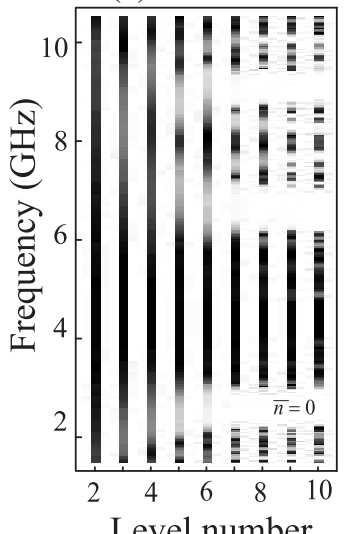

Level number (b) Thue-Morse

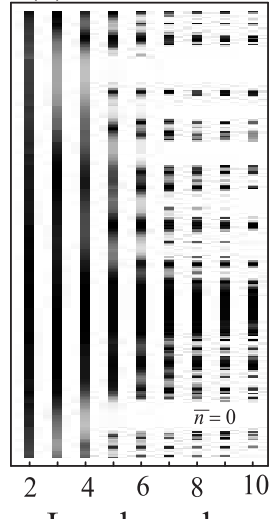

Level number (c)

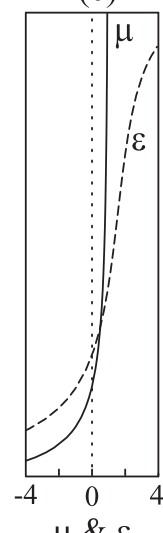

FIG. 2 Photonic spectra for the (a) Fibonacci and (b) Thue-Morse multilayers. The right figure shows the frequency behaviour of the constitutive parameters corresponding to the metamaterial layers.
Note the presence of a gap in the frequency range where the MM refractive index is negative $(f<3.133 \mathrm{GHz})$. The position and size of this gap become stabilized for $S>6$. In Ref. [20] it has been proved for the Fibonacci multilayer that this gap is a zero $-\bar{n}$ gap. The average index for the Sth Fibonacci level can be written as

$$
\bar{n}_{F I B}(S)=\frac{F_{S} d_{A} n_{A}+F_{S-1} d_{B} n_{B}}{F_{S} d_{A}+F_{S-1} d_{B}} .
$$

As it is well known, the basic mathematical relationship between the Fibonacci series and the golden ratio is given by the asymptotic limit

$$
\tau=\lim _{S \rightarrow \infty} \frac{F_{S}}{F_{S-1}}=\frac{1+\sqrt{5}}{2}
$$

from which we can easily find that $\bar{n}_{F I B}(S \rightarrow \infty)$ is zero at $2.547 \mathrm{GHz}$, a value lying in the lower gap shown in Figure 2a.

Taking into account that the Thue-Morse multilayer presents the same numbers of A-type and B-type slabs for a given level $S$, the average index does not depend on this parameter, being

$$
\bar{n}_{T-M}=\frac{d_{A} n_{A}+d_{B} n_{B}}{d_{A}+d_{B}} .
$$

Then, the average index of the system is zero at $2.288 \mathrm{GHz}$, and a gap does open at that frequency. It is instructive to note that the Thue-Morse multilayer can be understood as a periodic multilayer associated with period $\Lambda=d_{A}+d_{B}$, but with an 'A' and ' $\mathrm{B}$ ' exchange in some unit cells, so the zero- $\bar{n}$ gap arises around the same frequency values for both cases. The upper gaps in Figures $2 a$ and $b$ are based on standard interference mechanisms.

Now we study the influence of the incidence angle, $\theta$, on the photonic spectra of these aperiodic systems. At normal incidence, TE and TM polarization contributions are equivalent due to the symmetry of the structure. In an oblique incidence, however, the contributions of TE and TM polarizations are no longer equivalent. This fact is shown in Figure 3 where we compare the photonic spectra of the aperiodic multilayers considered for $\theta=45^{\circ}$.

As in the periodic case [17], we observe in Figure 3 the existence of two new band gaps around the frequencies where the effective parameters $\epsilon_{A}$ or $\mu_{A}$ are zero. These gaps are polarization-selective since the zero- $\mu$ gap appears for TE, but not for TM polarization, whereas the zero- $\epsilon$ gap appears for TM, but not for TE polarization. The size of these gaps becomes stabilized for $S>5$ in both aperiodic structures. Furthermore, zero- $\mu$ and zero- $\epsilon$ gaps are more robust than the zero- $\bar{n}$ gap. We observe that for $S<5$, the zero- $\bar{n}$ gap depth decreases significantly. However, the zero- $\mu$ and zero- $\epsilon$ gaps always appear, even for $S=2$. 

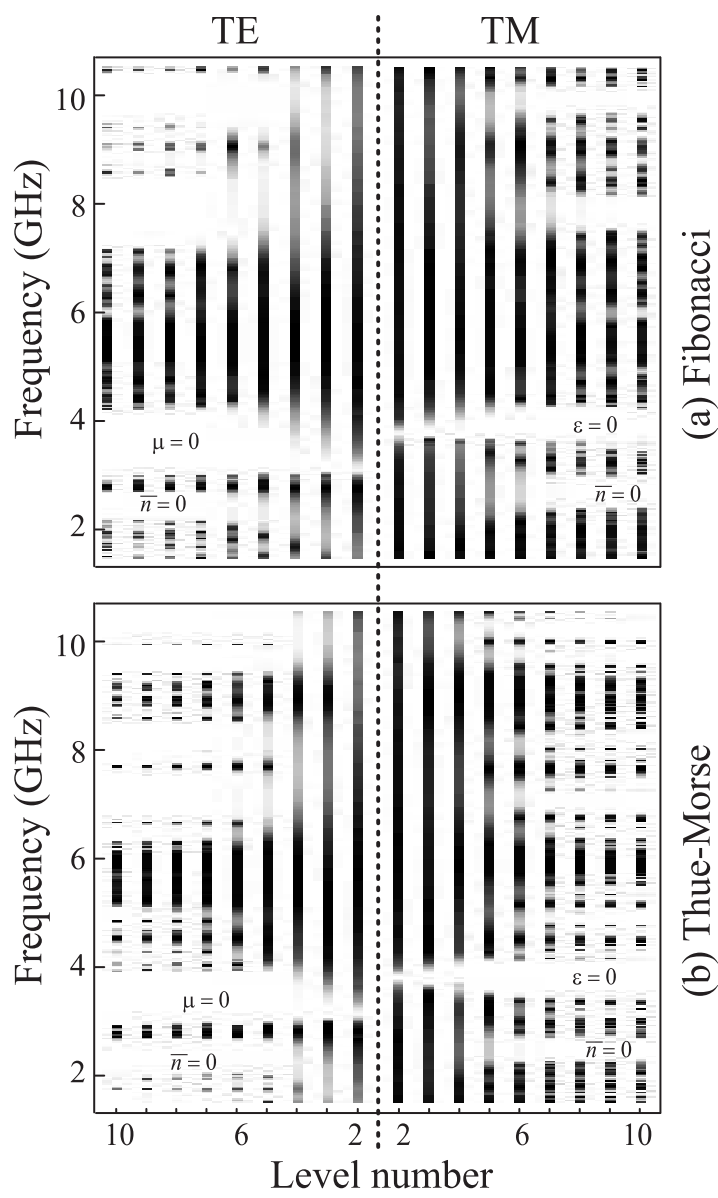

FIG. 3 Photonic spectra for the (a) Fibonacci and (b) Thue-Morse multilayers at oblique incidence $\left(\theta=45^{\circ}\right)$

It is worth noting that in any multilayer, either periodic or aperiodic, the central frequency of the zero- $\bar{n}$ gap depends on the ordering of the different building blocks, whereas the central frequencies of the zero- $\mu$ and zero- $\epsilon$ gaps are fixed by the condition that a constitutive parameter of the MM becomes zero. As a result, multilayers with identical building blocks but different spatial patterns may exhibit different degrees of interaction between zero $-\bar{n}$, zero $-\mu$ and zero $-\epsilon$ gaps. For the binary multilayers considered here, the degree of interaction can be controlled by tuning the values for the ratio $d_{B} / d_{A}$ between widths of air and MM layers. However, equations (8) and (9) show that this tuning effect may work differently for different spatial patterns: while the value of the central frequency for the zero- $\bar{n}$ gap in the Fibonacci asymptotic limit $(S \rightarrow \infty)$ involves the golden ratio, in the Thue-Morse case this value is identical to the periodic case.

In Figure 4 we plot the dependence of the photonic spectra of the aperiodic structures analyzed on the incident angle for TE and TM polarization. We have considered different generation levels of the systems in order to compare the results obtained with a similar numbers of layers.

The width of the zero- $\bar{n}$ gap does not change appreciably for TE polarization, but it gets narrower when $\theta \rightarrow 90^{\circ}$ for TM polarization, specially in the Thue-Morse structure. On the other hand, the new zero- $\mu$ and zero- $\epsilon$ gaps increase with the angle $\theta$. We observe a similar tendency with these non-Bragg gaps in both aperiodic structures, since it is the material dispersion of the MM what mainly determines their appearance in the photonic spectra. Additionally, since non-Bragg gaps are not based on interference mechanisms, they may remain invariant to scaling or disorder as in the periodic case [17].
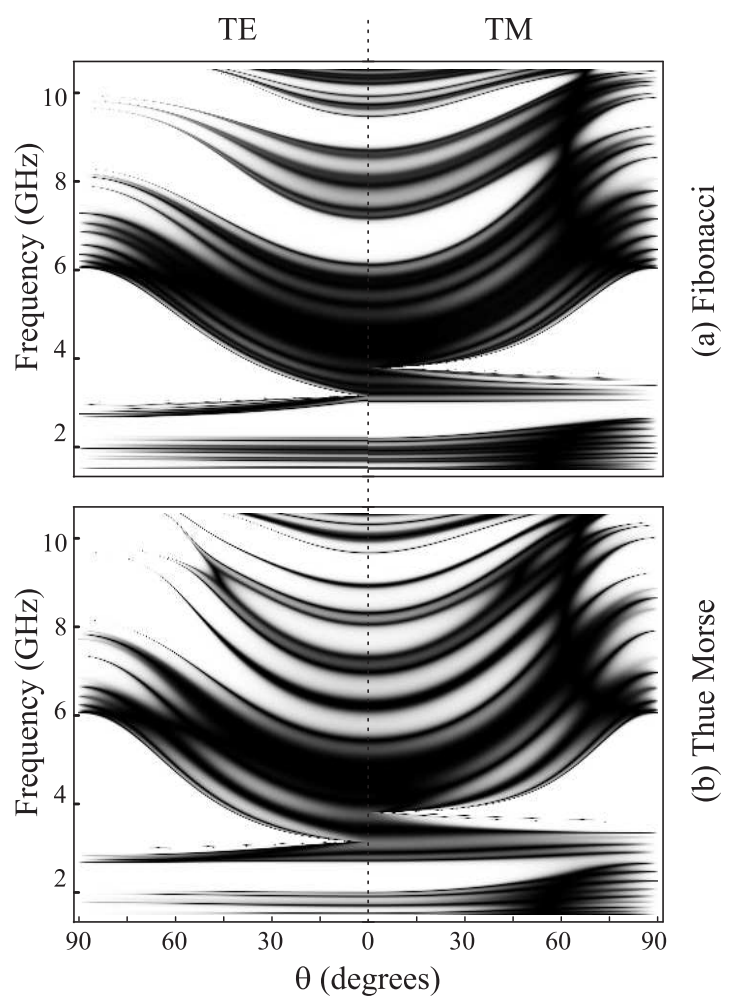

FIG. 4 Photonic spectra as a function of the incident angle for the (a) Fibonacci and (b) Thue-Morse multilayers generated at level numbers $S=8$ and $S=6$, respectively.

It should be noted that, although in practice MMs are microstructured systems, they have been modeled as continuous media in our simulations, since its microstructural features are assumed to be considerably smaller in size than the wavelength. The dispersion relations of the MM constitutive parameters, given in our case by Eq. (7), are at the base of the new properties found when we combine MMs and ordinary materials.

\section{C O N C L USION S}

Summarizing, the photonic spectra provided by 1D aperiodic multilayers -namely the Fibonacci and Thue-Morse latticescombining ordinary positive index materials and dispersive metamaterials have been examined. Using the conventional transfer matrix method, it was found that the non-Bragg band gaps exist for the aperiodic structures. The existence of the zero $-\bar{n}$ band gap has been proven previously in quasiperiodic (Fibonacci) multilayers. Here we have found this kind of gap in other aperiodic structures such as the Thue-Morse lattice. The proportion of MM and conventional material in the aperiodic multilayer determines the central frequency of the zero- $\bar{n}$ gap. Furthermore, for oblique incidence, we have shown that aperiodic multilayers containing MMs can also exhibit nonBragg band gaps near frequencies where either the magnetic permeability $\mu$ or the permittivity $\epsilon$ of the MM changes sign. These polarization-selective gaps are more robust than the 
zero $-\bar{n}$ gap. Even for lower values of the generation order of the aperiodic sequence, the zero- $\mu$ and zero- $\epsilon$ gaps appear in the photonic spectra. It is worth noting that the above results may be generalized to other aperiodic multilayers, like period-doubling or silver-mean lattices, among others.

\section{ACK N O W L E D G M N T S}

We acknowledge useful discussions with Prof. Walter D. Furlan. This work was funded by Plan Nacional I+D+i (grants TEC2005-07336-C02-02/MIC and TEC2004-04313-C02-02), Ministerio de Educación y Ciencia, Spain. RAD acknowledges partial support from Consejo Nacional de Investigaciones Científicas y Técnicas (CONICET) and Agencia Nacional de Promoción Científica y Tecnológica (ANPCYTBID802/OC-AR03-14099). JAM also acknowledges financial support from Programa de Incentivo a la Investigación de la UPV 2005, Vicerrectorado de Innovación y Desarrollo, Universidad Politécnica de Valencia, Spain.

\section{References}

[1] E. Yablonovitch, "Photonic band-gap structures" J. Opt. Soc. Am. B 10, 283-295 (1993).

[2] J. D. Joannopoulos, R. D. Meade, and J. N. Winn, Photonic Crystals: Modeling the Flow of Light (Princeton University Press, Priceton, 1995).

[3] Y. Fink, J. Winn, S. Fan, C. Chen, J. Michel, J. Joannopoulos, and E. Thomas, "A Dielectric Omnidirectional Reflector" Science 282, 1679-1682 (1998).

[4] D. S. Wiersma, P. Bartolini, A. Lagendijk, and R. Righini, "Localization of light in a disordered medium" Nature 390, 671-673 (1997).

[5] M. Bertolotti, P. Masciulli, and C. Sibilia, "Spectral Transmission properties of self-similar optical Fabry-Perot resonator" Opt. Lett. 19, 777-779 (1994).

[6] A. V. Lavrinenko, S. V. Zhukovsky, K. S. Sandomirski, and S. V. Gaponenko, "Scaling properties of an optical Cantor filter" Phys. Rev. E 65, 036621 (2002).

[7] D. L. Jaggard and X. Sun, "Reflection from fractal multilayers" 0pt. Lett. 15, 1428-1430 (1990).

[8] S.N. Zhu, Y.Y. Zhu, and N.B. Ming, "Quasi-phase-matched thirdharmonic generation in a quasi-periodic optical superlattice" Science 278, 843-846 (1997).
[9] X.B. Cai and X.F. Xuan, "Optical harmonic generation in a Fibonacci dielectric superlattice of $\mathrm{LiNbO}_{3}$ " Opt. Commun. 240, 227233 (2004).

[10] F. Qiu, R. W. Peng, X. Q. Huang, Y. M. Liu, M. Wang, A. Hu, and S. S. Jiang, "Resonant transmission and frequency trifurcation of light waves in Thue-Morse dielectric multilayers" Europhys. Lett. 63, 853-859 (2003).

[11] L. Moretti, I. Rea, L. Rotiroti, I. Rendina, G. Abbate, A. Marino, and L. De Stefano, "Photonic band gaps analysis of Thue-Morse multilayers made of porous silicon" Opt. Express 14, 6264-6272 (2006).

[12] E. Maciá, "The role of aperiodic order in science and technology" Rep. Prog. Phys. 69, 397-441 (2006).

[13] Z. Cheng, R. Savit, and R. Merlin, "Structure and electronic properties of Thue-Morse lattices" Phys. Rev. B 37, 4375-4382 (1998).

[14] V. G. Veselago, "The electromagnetics of substances with simultaneously negative $\epsilon$ and $\mu$ " Sov. Phys. Usp. 10, 509-514 (1968).

[15] D. Smith, J. Pendry, and M. Wiltshire, “Metamaterials and negative refractive index" Science 305, 788-792 (2004).

[16] J. Li, L. Zhou, C. T. Chan, and P. Sheng, "Photonic band gap from a stack of positive and negative index materials" Phys. Rev. Lett. 90, 083901 (2003).

[17] R. A. Depine, M. L. Martínez-Ricci, J. A. Monsoriu, E. Silvestre, and P. Andrés, "Zero permeability and zero permittivity band gaps in 1D metamaterial photonic crystals" arXiv: physics/0606069 (2006).

[18] J. Gerardin and A. Lakhtakia, "Spectral response of Cantor multilayers made of materials with negative refractive index" Phys. Lett. 301, 377-381 (2002).

[19] M. Maksimoviæ and Z. Jakšiæ, "Emittance and absorptance tailoring by negative refractive index metamaterial-based Cantor multilayers" J. Opt. A: Pure Appl. 0pt. 8, 355-362 (2006).

[20] J. Li, D. Zhao, and Z. Liu, "Zero- $\bar{n}$ photonic band gap in a quasiperiodic stacking of positive and negative refractive index materials" Phys. Lett. A 332, 461-468 (2004).

[21] H. He and W. Zhang, "Transmission spectra in symmetrical Fibonacci superlattices composed of positive and negative refractive index materials" Phys. Lett. A 351, 198-204 (2006).

[22] P. Yeh, A. Yariv, and C. Hong, "Electromagnetic propagation in periodic stratified media. I. Ceneral theory" J. Opt. Soc. Am. 67, 423-438 (1977).

[23] Yu Yuan, Lixin Ran, Jiangtao Huangfu, Hongsheng Chen, Linfang Shen and Jin $\mathrm{Au}$ Kong, "Experimental verification of zero order bandgap in a layered stack of left-handed and right-handed materials" Opt. Express 14 2220-2227 (2006). 